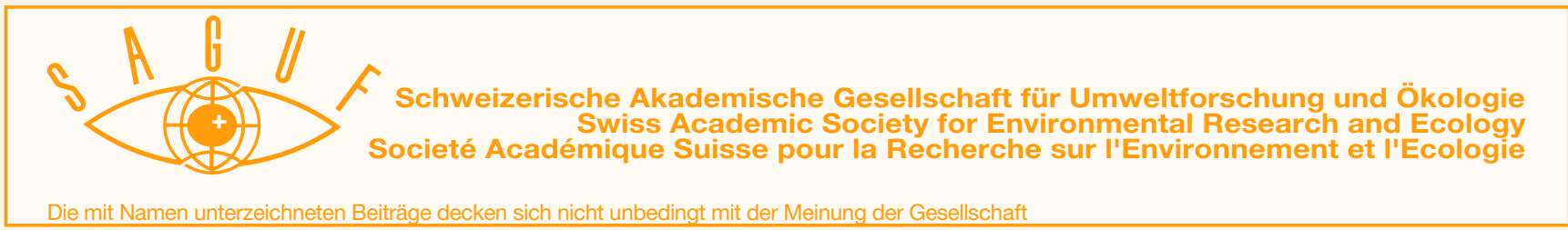

\title{
Umweltforschung im ETH-Bereich auf Innovation ausgerichtet
}

\begin{abstract}
Nach einer Phase des Wachstums ist die Umweltforschung im ETH-Bereich seit einigen Jahren einem erheblichen Veränderungsdruck ausgesetzt, der bereits zu einer neuen organisatorischen und teilweise auch inhaltlichen Ausrichtung geführt hat. Obwohl der Ausgang dieses Prozesses derzeit noch nicht absehbar ist, scheint die Bedeutung der Umweltforschung nicht gefährdet. Diese Einschätzung gründet auf der gesellschaftlichen Relevanz der Themen, der weiterhin steigenden Zahl von Studierenden und der internationalen Anerkennung der Qualität der bisher geleisteten Arbeit. Die wichtigste Herausforderung liegt im Abstimmen der unterschiedlichen Entwicklungen und dem Bemühen um eine gemeinsame Ausrichtung. Die derzeit oft postulierte "Konzentration der Kräfte" kann zu einer Stärkung der Umweltforschung führen, wenn eine Profilierung dieses Forschungszweiges als Ganzes gelingt.
\end{abstract}

Die Eidgenössischen Technischen Hochschulen in Zürich (ETHZ) und Lausanne (EPFL) bilden zusammen mit dem Paul-Scherrer-Institut (PSI), der Eidgenössischen Forschungsanstalt für Wald, Schnee und Landschaft (WSL), der Eidgenössischen Materialprüfungsund Forschungsanstalt (EMPA) und der Eidgenössischen Forschungsanstalt für Wasserversorgung, Abwasserreinigung und Gewässerschutz (EAWAG) den ETHBereich. Seine oberste Leitungsbehörde ist der ETH-Rat. An den beiden ETHs werden rund 17500 Studierende von mehr als 10300 Mitarbeitenden, davon rund 500 Professorinnen und Professoren, betreut. Alljährlich werden rund 1800 Diplome und mehr als 700 Doktorate verliehen. Die Forschungsanstalten leisten mit ihren 2800 Mitarbeiterinnen und Mitarbeitern einen zunehmend wichtigen Beitrag an die Aus- und Weiterbildung im ETH-Bereich und erbringen zusätzlich zur Forschung und Lehre wissenschaftliche und technische Dienstleistungen. Seit dem Jahr 2000 wird der ETH-Bereich mit Leistungsauftrag und Globalbudget geführt. Für die Periode

\section{Mich schmerzt die Situation der Umweltforschung}

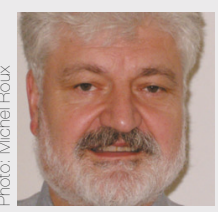

Interview mit Dr. Heinrich Neukomm, Leiter des Bereichs Wissenschaft im Generalsekretariat des ETH-Rats. Die Fragen stellte Michel Roux.

Ist Umweltforschung für den ETH-Rat gegenwärtig ein Thema? Mit dem Abschluß der strategischen Planung für die Jahre 2004-2007 sowie der Zielvereinbarungen zwischen dem ETH-Rat und den sechs Institutionen des ETH-Bereichs liegt die inhaltliche Verantwortung für die Umweltforschung ganz bei den ETHs und den vier Forschungsanstalten. Der ETH-Rat befaßt sich heute hauptsächlich mit der Ausgestaltung der Hochschullandschaft in der Schweiz nach 2008. Er engagiert sich aber auch sehr im Bereich Nachhaltigkeit.

Wie sehen Sie die Entwicklung der Umweltforschung an den einzelnen Institutionen? Da sehe ich einerseits Unterschiede zwischen der ETHZ und der EPFL aber auch zwischen den ETHs und den Forschungsanstalten. Die Schulleitung der ETHZ hat ihre international profilierte Umweltforschung durch die Neuordnung des sogenannt Grünen Bereichs grundsätzlich umgestaltet. An der EPFL ist nun vorgesehen, diesen Bereich gezielt zu stärken. Die geplante Berufung eines namhaften Umweltforschers von der Johns Hopkins University (Baltimore) betrachte ich als einen sehr klugen Schritt. Insgesamt gute Rahmenbedingungen für die Umweltforschung bieten meines Erachtens die Forschungsanstalten. Sie sind in den Kantonen besser verankert und eröffnen gute Möglichkeiten für interdisziplinäres Arbeiten, was für die Umweltforschung sehr wichtig ist.

Wo sehen Sie die größten Schwierigkeiten für die Umweltforschung im ETH-Bereich? Die Schwierigkeiten sind eigentlich bestens bekannt. Um an den Hochschulen wissenschaftlich Erfolg zu haben, müssen die Forscherinnen und Forscher disziplinär arbeiten. Das ist ein großes Handicap für die Umwelt- und Nachhaltigkeitsforschung. Sie ist mit Aufgaben konfrontiert, die multi-, inter- und transdisziplinär gelöst werden müssen. Und dafür verfügen wir noch über kein gutes Bewertungssystem.

Sie verstehen also, weshalb Wissenschaftlerinnen und Wissenschaftler recht gemischte Gefühle in Bezug auf die Bedeutung der Umweltforschung im ETH-Bereich haben?

Ja, sicher. Mich persönlich schmerzt es, daß die Umweltforschung im ETH-Bereich gegenwärtig nicht immer jene Anerkennung findet, die sie eigentlich verdient.

Was wäre Ihrer Meinung nach zu tun, um die Situation zu verbessern?

Es gibt im ETH-Bereich glücklicherweise zahlreiche ausgewiesene Wissenschaftlerinnen und Wissenschaftler, die trotz den schwierigen Rahmenbedingungen für inter- und transdisziplinäre Forschung innovative Umweltforschung betreiben. Diese Leaderfiguren sollten sich über die Grenzen der eigenen Disziplin und Institution stärker als bisher vernetzen und gemeinsam an Schwerpunkten arbeiten. Damit könnten sie der Umweltforschung sowohl nach innen als auch nach außen ein stärkeres Profil geben. Als gutes Beispiel einer erfolgreichen Zusammenarbeit zeichnet sich für mich immer mehr NOVATLANTIS ab - die gemeinsame Initiative der Forschungsanstalten und des ETH-Rates, um die Entwicklung und Verbreitung von nachhaltigen Technologien und Raumnutzungskonzepten dank einer guten Kooperation zwischen Wissenschaft und Praxis zu beschleunigen. Das Projekt ermöglicht besonders eine Vernetzung der sechs Institutionen des ETH-Bereichs mit der Universität Zürich, dem Kanton Zürich und Industriekreisen. 
2004-2007 umfaßt das vom Bund zur Verfügung gestellte Globalbudget 1957 Millionen CHF pro Jahr, rund 12 Prozent mehr als in der Vier-Jahres-Periode zuvor. Dennoch führen laufende Sparanstrengungen auf Bundesebene $\mathrm{zu}$ einer finanziell angespannten Lage, in der zudem von den Hochschulen und Forschungsanstalten ein verstärkter Beitrag zur Erhöhung der wirtschaftlichen Konkurrenzfähigkeit der Schweiz erwartet wird.

\section{Aufbau der Umweltforschung}

Im letzten Quartal des zwanzigsten Jahrhunderts hat der ETH-Bereich begonnen, sich der gesellschaftlichen Diskussion über Technik, Technologieentwicklung und ihren Folgen zu öffnen. Stichworte hierfür sind beispielsweise Überdüngung der Seen (Träger: EAWAG), Waldsterben (Träger: WSL) oder Kernenergie (Träger: PSI). In dieser Zeit ist es gelungen an den ETHs drei Bereiche in Forschung und Lehre aufzubauen (1) Umweltnaturwissenschaften, (2) Umweltingenieurwissenschaften und (3) Produktökologie (einschließlich Energiebereitstellung). Diese Entwicklung wurde maßgeblich von der ETHZ getragen, wobei ihre grundsätzlich breitere Ausrichtung und ihre enge Kooperation mit den Forschungsanstalten wesentlich zum Erfolg beitrugen. Das so entstandene Netzwerk ist verteilt auf verschiedene Departemente der Hochschulen sowie auf die Forschungsanstalten und wird durch die gemeinsame Lehrtätigkeit in umweltorientierten Studienrichtungen sowie Forschungskooperationen zusammen gehalten, unter anderem durch die Alliance for Global Sustainability (AGS). Rückblickend wird anerkannt, daß die ETHZ sich eine führende Rolle in der Umweltforschung erarbeitet hat, die es langfristig zu sichern und weiterzuentwickeln gilt.

Seit der Jahrtausendwende scheint dieser Prozeß der Öffnung abgeschlossen. Die ETHs und einige der Forschungsanstalten besinnen sich auf ihre traditionell technik- und disziplinär orientierten Kernbereiche zurück. Die Wachstumsphase der Umweltforschung scheint damit abgeschlossen.

\section{Reorganisation und Verunsicherung}

Gleichzeitig sehen sich die Leistungsträger, die Forschung und Lehre im Umweltbereich tragen, einem erheblichen Veränderungsdruck ausgesetzt. Im Falle der ETHs gibt es hierfür zwei Auslöser. Einerseits sind es Initiativen der Schulleitungen, die darauf zielen, neue, umfassendere und schlagkräftige Organisa-

\section{Fachhochschulen der Nachhaltigkeit verpflichtet}

Die neuen Fachhochschulen haben ihren Fokus von der Umweltforschung hin zur Nachhaltigkeit gewechselt: Die Zürcher Hochschule betreibt von ihrem Zentrum Sustainability ausgehend ein Netzwerk für die regionale nachhaltige Entwicklung.

Der Gedanke, Umweltschäden zu reparieren und weiteren Raubbau an den natürlichen Ressourcen verhindern zu können, beseelte die Aktivitäten der Umweltforscher an den höheren Technischen Lehranstalten (HTL) der 1980er und 1990er Jahre. Wertvolle Beiträge zu technischen Lösungen in der Kontrolle heikler Prozesse, wie in der Fertigung und Entsorgung von Gütern oder in der Gewinnung und Nutzung von Energie, konnten effizienter gestaltet werden.

Mit dem Umbau und der Aufwertung der HTL und der Höheren Wirtschafts- und Verwaltungsschulen (HWV) zu Fachhochschulen (FH) und den vielerorts als Fusionsprodukte neu entstandenen Mehrsparten-FH (zum Beispiel Architektur, Technik, Wirtschaft und Kommunikation zur Zürcher Hochschule Winterthur (ZHW) oder Technik, Wirtschaft und Soziale Arbeit in St. Gallen zur FHSG), haben sich in den letzten fünf Jahren die Schwerpunkte der Forschungsaktivitäten merklich verschoben. Nach wie vor werden technische Systemlösungen erarbeitet, aber die Nähe von Wirtschafts- und Sozialwissenschaften zu den Natur- und Ingenieurwissenschaften haben zu neuen interdisziplinären Forschungsgemeinschaften geführt, die sich unter der Leididee der nachhaltigen Entwicklung entsprechend breiter orientieren. Der Entwurf zum neuen Fachhochschulgesetz bietet dazu die rechtliche Grundlage, in dem es die FH auf die Förderung einer nachhaltigen Entwicklung verpflichten will.

\section{Zentrum Sustainability zsa-ZHW}

Die ZHW hat diesen staatlichen Auftrag schon vorweg umgesetzt. Sie gründete im Sommer 2003 das "Zentrum Sustainability zsa-ZHW". Dieses vereint Wissenschaftlerinnen und Wissenschaftler der Umweltnaturwissenschaften, Biologie, Geographie, Ökonomie, Soziologie und Geisteswissenschaften unter einem Dach. Projekte und Forschungsaufträge aus den Themen Umweltmanagementsysteme (UMS), Social Management Systems (SMS), nachhaltige Planung in Städten und Gemeinden, neue finanzielle Unterstützungsformen für Berggebiete, werden im zsa-ZHW in interdisziplinär besetzten Teams erarbeitet. Das zsa-ZHW bildet mit weiteren Departementen der ZHW und externen Partnern in Universitäts- und Fachhochschulinstituten sowie in privaten Unternehmungen ein Netzwerk, das in dieser Konstellation als Kompetenzverbund für die regionale nachhaltige Entwicklung wirkt.

Die Aufträge des zsa-ZHW werden durch den Schweizerischen Nationalfonds (SNF), die Kommission für Technologie und Innovation (KTI), von Stiftungen, durch kantonale Beiträge und hauptsächlich von den jeweiligen Partnern aus Wirtschaft und öffentlicher Hand alimentiert. Sie reichen von traditioneller Grundlagenforschung über Methoden- und Instrumentenerarbeitung bis hin zu eigentlichen Dienstleistungen für die Auftraggeber.

\section{Ausblick}

Den Aufgabenstellungen der FH entsprechend findet sich die Umweltforschung in einer neuen Position. Sie ist nicht mehr Aufgabe für sich, sondern Teil der umfassenden Sichtweise und Problemlösungsstrategie "Sustainability". Damit hat sich die Umweltforschung an den FH weg bewegt vom Fokus der Reparaturleistungen hinein in die zukunftsträchtige Dimension zur Unterstützung einer nachhaltigen Vorsorgepolitik. Somit ist sie gut ausgerichtet, um auch Innovationskooperationen mit dem ETH-Bereich einzugehen.

Erich Renner Leiter Zentrum Sustainability zsa-ZHW

tionseinheiten zu schaffen, in denen die Umweltforschung integriert wird. Anderseits führt das neue Bachelor/Mastersystem zu Veränderungen in den umweltorientierten Studiengängen. Diese Veränderungen führten $\mathrm{zu}$ folgenden neuen Organisationseinheiten:

- Zentrum für Umwelt und Natürliche Ressourcen (ZUNR/ETHZ): Mit Schaffung dieses Zentrums (Gründung 2005) sollen die systemorientierten Naturwissenschaften (Umweltnatur-, Forst-, Erdund Agrar- sowie Lebensmittelwissen- schaften) strukturell koordiniert sowie nach innen und außen gestärkt werden. Damit einher geht die Zusammenlegung der Forst- und Umweltnaturwissenschaften im Departement Umweltwissenschaften (2004), gemeinsam gestaltete Bachelorstudiengänge in den systemorientierten Naturwissenschaften sowie die Entwicklung von Masterstudiengängen. Damit werden systemorientierte Ansätze zur Untersuchung abiotischer, biotischer und anthropogener Prozesse auf allen Skalen angestrebt, ergänzt um Ziel- und um 
Transformationswissen zum Management von Umweltproblemen.

- Erneuerung der raumbezogenen Wissenschaften am Departement für Bau, Umwelt und Geomatik (D-BAUG/ETHZ): In diesem Zusammenhang $\mathrm{zu}$ nennen sind die Neugründung des Instituts für Raum- und Landschaftentwicklung (IRL) und die Umbenennung des Instituts für Verkehrsplanung (neu: Verkehrsplanung statt -technik) als Teile des Netzwerks Stadt und Landschaft (Nachfolgeorganisation des Instituts für Orts-, Raumund Landesplanung) sowie das Schaffen eines Netzwerkes für auf Naturgefahren bezogene Forschung. Gleichzeitig wird die Stärkung der Umwelt- und Geomatikingenieurwissenschaften diskutiert.

- Im Zuge einer generellen Neuorganisation der EPFL wurden die ehemaligen Departemente "génie rural", "génie civil" und "architecture" zu einer neuen Fakultät für Environement Naturel, Architectural et Construit (ENAC) zusammengelegt (Gründung 2002). Dies war verbunden mit einer Umbenennung der Kultur- in Umweltingenieurwissenschaften und einer Reform des Studiengangs, in der die Umweltwissenschaften eine gröBere Bedeutung im Lehrplan erhalten. Gleichzeitig bemüht sich die EPFL um eine stärkere Zusammenarbeit mit den Forschungsanstalten.

Auch an den Forschungsanstalten finden derzeit tiefgreifende organisatorische und inhaltliche Veränderungen statt. - Am PSI und an der EMPA wird die Suche nach neuen Lösungen für die Energie- und Gebäudetechnik sowie für Fahrzeugtechnologien der 2000-Watt-Gesellschaft intensiviert. In diesen Praxisfeldern stehen die Erhöhung der Energieund Ressourceneffizienz, das "Life Cycle Engineering" und die Nutzung von erneuerbaren Energien im Vordergrund (zum Beispiel im Projekt ECOINVENT, das von der EMPA, dem PSI und den beiden ETHs getragen wird). In diesem Zusammenhang ist auch das EMPA-Programm "Sustainability in the Information Society" zu nennen, in dem ein internationales Kompetenzzentrum für Nachhaltigkeitsforschung in der Informationsgesellschaft aufgebaut werden soll.

Die EAWAG befaßt sich mit dem Wasser als einem Schlüsselfaktor für Leben und für intakte Ökosysteme. Sie übt eine weltweit anerkannte wissenschaftliche Führungsrolle bei der Förderung eines nachhaltigen Umgangs mit Wasser aus. Diese soll weiter ausgebaut werden, indem auch die EAWAG die sozialwissenschaftlichen Kompetenzen im Bereich der Umwelt- und Ressourcenökonomie verstärken will.
- Die WSL entwickelt sich zu einem Umweltforschungszentrum, das wissenschaftliche Grundlagen in Form von Daten, Modellen und Managementsystemen für eine nachhaltige Nutzung und Gestaltung der Landschaft entwickelt. $\mathrm{Zu}$ diesem Zweck wird auch ein vertieftes Verständnis der ökonomischen und sozialen Systeme aufgebaut. Dieses Wissen soll zukünftig verstärkt in die Handlungsfelder der Raumentwicklung (Raumplanung, Regionalentwicklung) eingebracht werden. Das Innovationsund Kooperationsprojekt "Landschaft im Ballungsraum" wirkt hier schon recht erfolgreich als Wegbereiter. Ein zweites Engagement der WSL gilt dem Mitgestalten eines neuen Umgangs mit Naturgefahren und - damit verbunden dem Aufbau eines integralen Risikomanagements, das neben den Naturgefahren auch die technologischen, ökologischen und gesellschaftliche Risiken umfassen soll.

Allen Initiativen ist das Bemühen um eine stärkere Zusammenarbeit mit Sozialwissenschaften und Ökonomie gemein: durch "fachfremde" Professuren in den jeweiligen Institutionen einerseits und Kooperationen mit anderen Departementen und den Universitäten anderseits. Die ETHs sind in dieser veränderten Konstellation grundsätzlich in allen Schwerpunktbereichen für die Umweltforschung im Hochschulbereich vertreten, allerdings ist die Koordination der verschiedenen Aktivitäten unzureichend, teilweise fehlen auch noch Professuren, und in einigen Bereichen fehlen klare Profile der verschiedenen Institutionen in Abgrenzung zueinander. Gründe dafür liegen in der Aktualität der geschilderten Entwicklungen, in denen die verschiedenen Institutionen primär mit sich selbst beschäftigt waren.

Zusätzlich erschwert wurden Koordinationsbemühungen durch Verlustäng- ste, die angesichts der Schwerpunktsetzung der Schulleitungen und der Tragweite der organisatorischen Veränderungen verständlich sind. Für die Beteiligten aller Institutionen stellte sich implizit die Frage nach den Gewinnern und Verlierern der aktuellen Entwicklungen. Das drückt auf die Stimmung.

\section{Aktuelle Entwicklung und Ausblick}

Dem allgemeinen Gefühl der Verunsicherung der letzten Jahre zum Trotz läßt sich eine erste positive Bilanz aus den Veränderungen ziehen. Die Reorganisationsprozesse kommen voran. Es ist zu hoffen, daß die Umweltforschung im ETH-Bereich tatsächlich gestärkt aus ihnen hervorgeht. Beispiele sind (a) die Umbenennung und Neuorientierung der Umweltingenieurwissenschaften an der EPFL und (b) die Aufnahme des Zentrums für Umwelt und Natürliche Ressourcen sowie der Themenbereiche "Raum und Infrastruktur" und "Energy Engineering Sciences" in die Liste der Strategische Erfolgspositionen der ETHZ. Die Zahl der Studierenden im Umweltbereich konnte auf hohem Niveau gehalten und teilweise noch gesteigert werden. Internationale Forschungskooperationen wie die AGS wurden in ihrer Bedeutung für die ETHs bestätigt und Initiativen der Forschungsanstalten wie ECOINVENT und NOVATLANTIS zeigen erste Früchte. Die Umweltforschung ist so heute ein wichtiger Leistungsträger des ETH-Bereichs, der sich durch seine Fähigkeit zu interdisziplinärer Forschung auszeichnet. In ihren Ansätzen zur problembezogenen Integration von Natur-, Ingenieur- und Sozialwissenschaften gilt die Umweltforschung als innovativ, was ihr in einigen der geschilderten Veränderungsprozesse die "Katalysator-Rolle" eingebracht hat. Solange jedoch Anreizsysteme der Wis-

\section{NOVATLANTIS und das Projekt "2000 Watt Gesellschaft"}

NOVATLANTIS ist bisher die bedeutendste gemeinsame Initiative der Forschungsanstalten des ETH-Bereichs. novatlantis setzt die neusten Erkenntnisse und Resultate aus der Forschung für eine nachhaltige Entwicklung von Ballungsräumen um. In Zusammenarbeit mit Behörden und Unternehmen werden Projekte realisiert, die gesellschaftliche und technische Aspekte der Nachhaltigkeit berücksichtigen. Mit dem Projekt "2000 Watt Gesellschaft" strebt NOVATLANTIS die schrittweise Realisierung einer Lebens- und Wirtschaftsform an, die mit einem Drittel des heutigen Energieverbrauchs auskommt und gleichzeitig die Lebensqualität verbessert. In der Pilotregion Basel ist inzwischen ein Praxislabor entstanden, wo im Rahmen der Stadtentwicklung die politische und ökonomische Machbarkeit einer nachhaltigen Gesellschaft ausgelotet werden.

Hinweis: Sie können das Weißbuch zur "2000 Watt Gesellschaft" bestellen unter WWW.NOVATLANTIS.ch. 
senschaft nach wie vor ausschließlich disziplinäre Leistungen bevorzugen (siehe Interview mit Heinrich Neukomm), ist zu befürchten, daß dieses Innovationspotential nicht voll ausgeschöpft werden kann. In dieser Situation droht möglicherweise der Rückfall in ein altes Verständnis von Innovation, das einseitig an neuen technischen Möglichkeiten mit hohem wirtschaftlichem Verwertungspotential orientiert ist und dabei die langfristigen Folgen für Natur, Gesellschaft und Wirtschaft außer acht läßt.

\section{Schlußfolgerungen}

(1) Im ETH-Bereich entwickelt sich eine Umweltforschung, die auf fundamentale Innovationen ausgerichtet wird, mit einem hohen Potential, nicht nur die Umweltsituation weltweit zu verbessern, sondern auch die wirtschaftliche Konkurrenzfähigkeit der Schweiz zu erhöhen. (2) Viele Kräfte der Träger der Umweltforschung im ETH-Bereich sind in Reorganisationsprozessen gebunden, $\mathrm{zu}$ hoch scheinen die Hürden, die sich einer produktiven Zusammenarbeit zwischen den Disziplinen sowie den Wissenschaftlerinnen und Wissenschaftlern in den verschiedenen Institutionen in den Weg stellen.

(3) Die interne und externe Kommunikation über Potentiale, Schwerpunkte und Modellvorhaben, getragen von den herausragenden Trägern der Umweltforschung im ETH-Bereich, könnten entgegen dem Zeitgeist - zeigen, daß Umweltforschung, die sich an den großen gesellschaftlichen Herausforderungen orientiert, nach wie vor von höchster strategischer Bedeutung ist.

(4) Gleichzeitig sind die Anreizstrukturen und damit auch die Leistungsvereinbarungen so zu verändern, daß die Leistungen der inter- und transdisziplinären Forschung gegenüber heute besser honoriert werden.

(5) Die Herausforderung der nächsten Jahre liegt insbesondere in der Fähigkeit zur Koordination und Zusammenarbeit zwischen den verschiedenen Institutionen im ETH-Bereich (insbesondere ZUNR,
D-BAUG, ENAC und die in der Umweltforschung tätigen Bereiche von WSL, PSI, EMPA und EAWAG). Nur wenn es gelingt, bestehende Verlustängste $\mathrm{zu}$ überwinden kann man an der notwendigen Neuorientierung der gesamten Umweltforschung an den Technischen Hochschulen arbeiten und so ihren nachhaltigen Erfolg sicher stellen.

Im Namen des SAGUF-Vorstands

Susanne Kytzia

Institut für Raum- und Landschaftsentwicklung, ETH Zürich

Michel Roux

Leiter Abteilung Kommunikation und Marketing, WSL Birmensdorf
Kontaktadresse SAGUF:
SAGUF-Geschäftsstelle
c/o Claude Théato
ETH Zürich HAD
CH-8092 Zürich
Telephon: (+41 1) 6326310
Telefax: (+41 1) 6321029
E-Mail: saguf@env.ethz.ch
http://www.saguf.unibe.ch

\section{Mitteilungen der Deutschen Gesellschaft für Humanökologie (DGH)}

Die mit Namen unterzeichneten Beiträge decken sich nicht unbedingt mit der Meinung der Gesellschaft

\section{UN-Dekade "Bildung für Nachhaltige Entwicklung 2005-2014"}

\section{Bildung als Instrument einer umfassenden Nachhaltigkeitspolitik}

Bereits in der Agenda 21, dem Aktionsplan für eine nachhaltige Entwicklung im 21. Jahrhundert, der bei der UNCED (UN-Konferenz für Umwelt und Entwicklung) in Rio de Janeiro 1992 verabschiedet wurde, war dem Thema Bildung ein wichtiges Kapitel gewidmet. Weltweit hat dies vielfältige Aktivitäten ausgelöst, die jedoch in der Regel drei Nachteile hatten: Erstens konzentrierten sich Bildungsanstrengungen fast ausschließlich auf die Schule, also auf Ausschnitte aus dem formalen Bildungssystem, die vornehmlich auf Kinder und Jugendliche als Zielgruppe begrenzt sind. Zweitens beschränkten sie sich überwiegend auf Umweltbildung, stellten also die ökologische Dimension der Nachhaltigkeit in den Vordergrund. Drittens hat Bildung als ein Instrument von Nachhaltigkeitspolitik unter mehreren noch nicht den Stellenwert erreichen können, der ihr zukommen müßte. In den großen Konventionen oder Protokollen (etwa zum Klimaschutz, zum Erhalt biologischer Vielfalt, zur Verminderung der Wüstenbildung) kommt "Bildung" zwar auch vor, aber meist in sehr allgemeiner Form als "Aufklärung der Öffentlichkeit", "Bewußtseinsbildung". So hat das Thema "Bildung" bei den internationalen Folgekonferenzen bisher kaum Gewicht oder Sichtbarkeit gewinnen können.

\section{Nachhaltigkeit als umfassendes Lernprogramm}

Erst 10 Jahre nach Rio, auf dem "Weltgipfel für Nachhaltige Entwicklung" in Johannesburg 2002, wurde erneut die besondere Bedeutung von Bildung und lebenslangem Lernen für die Förderung einer nachhaltigen Ent- wicklung hervorgehoben. Auf Empfehlung des Weltgipfels hat die Vollversammlung der Vereinten Nationen im Dezember 2002 die Dekade Education for Sustainable Development (UNDESD) für den Zeitraum 2005 bis 2014 ausgerufen. Mit der Koordination dieser Dekade wurde die UNESCO beauftragt. Alle Mitgliedstaaten der Vereinten Nationen sind aufgerufen, national und international Bildungsaktivitäten zu entwickeln, die das Ziel der Weltgesellschaft, die Lebens- und Überlebensbedingungen für die jetzt lebenden und die zukünftigen Generationen zu bewahren, unterstützen und Wege dorthin aufzeigen können.

Damit wird erkannt und anerkannt, daß nachhaltige Entwicklung ein langfristig angelegtes Gestaltungs-Programm ist, in dem immer wieder neu Ziele und Strategien erarbeitet werden müssen, die es möglich machen, innerhalb eines Handlungsraumes zu agieren, der durch "Leitplanken" von der Sphäre der NichtNachhaltigkeit getrennt ist. Nachhaltige Entwicklung bedeutet gezielte Veränderung nicht-nachhaltiger Lebensstile, und das impliziert ein umfassendes Lernprogramm, in dem die Notwendigkeiten von Natur- und Ressourcenschutz, die wirtschaftlichen Interessen der Menschen und die Gebote Verteilungsgerechtigkeit und Chancengleichheit der Generationen im Zusammenhang gesehen werden. 